\title{
Hadronic Interactions
}

\author{
Takeshi Yamazaki* \\ Faculty of Pure and Applied Sciences, University of Tsukuba, Tsukuba, Ibaraki 305-8571, Japan \\ Center for Computational Sciences, University of Tsukuba, Tsukuba, Ibaraki 305-8577, Japan \\ RIKEN Advanced Institute for Computational Science, Kobe, Hyogo 650-0047, Japan \\ E-mail: yamazaki@het.ph.tsukuba.ac.jp
}

\begin{abstract}
Understanding hadronic interactions is crucial for investigating the properties of unstable hadrons, since measuring physical quantities for unstable hadrons including the resonance mass and decay width requires simultaneous calculations of final scattering states. Recent studies of hadronic scatterings and decays are reviewed from this point of view.

The nuceon-nucleon and multi-nucleon interactions are very important to understand the formation of nucleus from the first principle of QCD. These interactions have been studied mainly by two methods, due originally to Lüscher and to HALQCD. The results obtained from the two methods are compared in three channels, $I=2$ two-pion, H-dibaryon, and two-nucleon channels. So far the results from the two methods for the two-nucleon channels are different even at the level of the presence or absence of bound states. We then discuss possible uncertainties in each method.
\end{abstract}

Recent results on the binding energy for helium nuclei are also reviewed.

The 32nd International Symposium on Lattice Field Theory

23-28 June, 2014

Columbia University New York, NY

${ }^{*}$ Speaker. 


\section{Introduction}

The ultimate goal of lattice QCD is to understand the properties of all hadrons quantitatively by first principle calculations. For stable hadrons, recent lattice QCD calculations have reached the level of a precise measurement of physical quantities such as mass and decay constant, thus touching the goal. On the other hand, we are far from this goal for unstable hadrons. The calculations and analyses involved are much more difficult and complex, because one has to simultaneously treat final scattering states to measure physical quantities of unstable hadrons. To calculate physical quantities in the scattering and decay processes, it is important to understand the interactions between hadrons based on QCD.

The most famous of hadronic interactions is the nuclear force, which binds nucleons into nuclei. Thus, another goal of the lattice QCD is to prove the formation of nucleus from the degrees of freedom of quarks and gluons.

For those goals, so far various studies have been carried out. In this report, recent studies on hadronic scattering, decay, and light nuclei are reviewed. Furthermore, it is reviewed consistency and inconsistency of results obtained from Lüscher's method and HALQCD methods in $I=2$ twopion, H-dibaryon, and two-nucleon channels. In the two-nucleon channels, possible systematic uncertainties in each method are discussed.

The organization of this report is as follows. In the next section, Lüscher's method is briefly reviewed, which allows one to evaluate the scattering phase shift of two particles from finite volume calculations. In Secs. 3 and 4 some recent studies for the scattering length and physical quantities in decay processes are presented, respectively. Sec. 5 presents a comparison of results from the Lüscher's method and HALQCD method, the latter having been recently proposed to calculate the potential between two hadrons, and a discussion on the uncertainties in each method. In Sec. 6 we review recent calculations of light nuclei and related studies. Conclusions are given in Sec 7.

\section{Lüscher's finite volume formula}

Lüscher's formula [1,2] in the original form enables to evaluate the scattering phase shift $\delta(p)$ of a two-particle system in the center-of-mass frame from the energy of two-particle state in finite volume, e.g., in the $\mathrm{S}$-wave case,

$$
\tan \delta(p)=\frac{\pi^{3 / 2} q}{Z_{00}\left(1 ; q^{2}\right)}
$$

where $q=L p /(2 \pi)$ and

$$
Z_{00}\left(s ; q^{2}\right)=\frac{1}{\sqrt{4 \pi}} \sum_{\vec{n} \in Z^{3}} \frac{1}{\left(\vec{n}^{2}-q^{2}\right)^{s}} .
$$

The relative momentum between the two particles, $p$, is determined from the two-particle energy, $W^{2}=4\left(m^{2}+p^{2}\right)$ with $m$ being mass of the particle. An important assumption is that the interaction of the two particles does not depend on volume, and that the interaction is well localized, i.e., there is a region such that $V(\vec{r}) \approx 0$ for $r<L / 2$ with $L$ being the linear spatial extent.

An extension of Lüscher's formula to moving frame, where the total momentum of two particles is non-zero, was first proposed by Rummukainen and Gottlieb [3]. Their result was confirmed by different derivations $[4,5]$, and extensions in moving frame with different total mo- 
menta, and several irreducible representations have also been derived [6, 7]. In the recent papers $[8,9,10,11,12]$ extensions of the formula in moving frames to the case of particles with different masses are derived, in which case mixings occur between even and odd partial waves. Those extensions are also reviewed by other plenary speakers [13, 14].

Lüscher's formula encompasses the case of a bound state as a pure imaginary solution for the relative momenta $p$, and indicates that the size dependence of the energy shift of the ground twoparticle state relative to the free two-particle state is exponentially small $[15,16]$. With this result as a background, study of size dependence of the energy shift has been employed in a number of recent papers to examine bound state formation in multi-hadron systems [17, 18, 19, 20, 21]. We shall call the methodology in these studies generally as Lüscher's method.

\section{Scattering length}

The scattering length $a_{0}$ is the simplest physical quantity in an S-wave scattering process, which is defined from the phase shift $\delta(p)$ by $a_{0}=\lim _{p \rightarrow 0}[\tan \delta(p) / p]$ in the zero relative momentum limit.

\section{1 $I=2$ two-pion channel}

The $I=2$ two-pion scattering is a very simple process involving no bound states or resonances in the low energy region. It is also the simplest from lattice calculation point of view, where only the direct and cross diagrams are necessary for the two-pion correlation function. While there have been various quenched calculations of $a_{0}$ in this channel, only dynamical calculations are reviewed in this report.

The left panel in Fig. 1 shows the results for $a_{0} m_{\pi}$ from dynamical calculations [22, 23, 24, 25, 26, 27, 28, 29, 30] together with two experimental results by E865 [31] and NA48/2 [32] plotted with burst symbols. The calculations by Hadron Spectrum [33, 7] and NPLQCD [34] Collaborations in $N_{f}=2+1$ full QCD are not plotted here, because the pion decay constant has not been calculated in the simulation parameter. The lattice results are compared with LO (leading order) ChPT (chiral perturbation theory) formula, $a_{0} m_{\pi}=-m_{\pi}^{2} /\left(8 \pi f_{\pi}^{2}\right)$, drawn by the dashed curve, where $f_{\pi}$ is the pion decay constant with the normalization of $f_{\pi}=132 \mathrm{MeV}$ at the physical pion mass. Most of lattice results agree with the formula. In the small $m_{\pi} / f_{\pi}$ region, however, partially quenched staggered (open square) [28] and nonperturbatively improved Wilson (closed circle) [30] results significantly deviate from LO ChPT.

The reason for the deviation of the staggered calculation [28] is not clear. The lightest data in Ref. [28] (open square) should agree with the one at the same $m_{\pi} / f_{\pi}$ in Ref. [29] (closed square), because their actions and simulation parameters are the same. On the other hand, the deviation of the improved Wilson calculation is probably due to chiral symmetry breaking effect of the Wilson quark action at non-zero lattice spacings [30].

The lattice results for $a_{0}$ at the physical $m_{\pi}$ are shown in the right panel of Fig. 1. For the chiral extrapolation of $a_{0}$, NLO (next-to-leading order) ChPT formula is employed in almost all calculations [23, 26, 28, 29]. On the other hand, Refs. [24, 30] use variations of NLO ChPT formula including systematic errors coming from finite lattice spacings. The value of Ref. [34] is 
estimated using the low energy constants extracted from $\delta(p)$ at $m_{\pi}=390 \mathrm{MeV}$ with NLO ChPT formula for the scattering amplitude, while they use the value of $f_{\pi}$ calculated with different action.

The lattice results are compared with the phenomenological determination, marked "CGL", obtained with NNLO ChPT [35] and the experimental results [32, 31] with and without ChPT constraint. Since in ChPT the scattering lengths for the $I=0$ and 2 channels share a common low energy constant, the error becomes smaller in the case with the constraint. Some lattice results have smaller error, even if including the systematic error, than the phenomenological determination and the experimental results. Therefore, $a_{0}$ calculation in the $I=2$ two-pion channel enters precision measurement era, where lattice result will be confirmed by experiment. In this conference, preliminary result of $N_{f}=2+1+1$ twisted mass quark calculation [36] is reported, which will calculate more precise value of $a_{0}$ at the physical $m_{\pi}$.
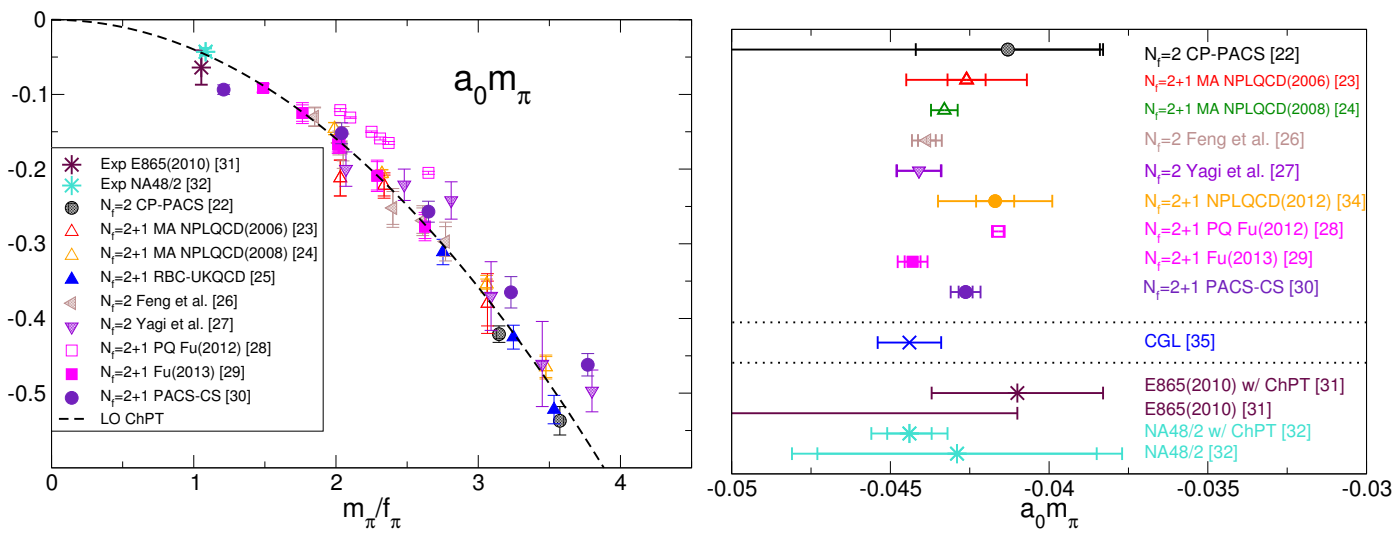

Figure 1: Left panel: $a_{0}$ in the $I=2$ two-pion channel calculated in full lattice QCD as a function of $m_{\pi} / f_{\pi}$, together with the prediction of LO ChPT (dashed curve). Right panel: values at physical $m_{\pi}$. Circle, up triangle, left triangle, down triangle, and square symbols denote results obtained with clover, domain wall, twisted mass, overlap, and improved staggered quark actions for valence quark, respectively. MA and PQ express mixed action with asqtad sea quark and partially quenched calculation. The experimental result by E865 [31] and NA48/2 [32], and the phenomenological determination using NNLO ChPT [35] (denoted by CGL in the right panel) are also shown.

\section{2 $I=1 / 2 K \pi$ channel}

The S-wave scattering in the $I=1 / 2 K \pi$ channel is more complicated than the $I=2$ twopion channel; it is in fact much more interesting because of the expected presence of $\kappa$ meson from phenomenology in this channel. In the region of heavy quark mass, $\kappa$ meson is indeed a bound state and so has to be taken into account in the $a_{0}$ calculation of $K \pi$ scattering [30]. In the case, one needs to calculate the first excited state in the system to evaluate $a_{0}$. Furthermore, for the calculation of $K \pi$ correlation function, we need to include the rectangular diagram, for which specific calculation methods are required, for example the stochastic LapH method [37] or the trapezoid diagram calculation method [38].

Due to these difficulties, there have been only four studies in the $I=1 / 2 K \pi$ channel; $N_{f}=0$ calculation by Nagata et al. [39], partially quenched $N_{f}=2+1$ by Fu [40], $N_{f}=2$ by Lang et al. [41], and $N_{f}=2+1$ by PACS-CS Collaboration [30]. The left panel of Fig. 2 shows the result 
of the four studies for $a_{0} m_{\pi}$, together with phenomenological determination [42]. The lattice data do not show a common $m_{\pi}^{2}$ dependence in contrast to the situation with the $I=2$ two-pion channel in Fig. 1. This is presumably due to the systematic error inherent in each calculations, e.g., a small spatial size of $\sim 2 \mathrm{fm}$ in the calculation of Ref. [41] and chiral symmetry breaking effect at the lightest data point of Ref. [30].

Similar to the calculations with the $I=2$ two-pion channel, $a_{0} m_{\pi}$ at the physical quark mass is estimated using NLO ChPT formula in Ref. [40] and its extension with chiral symmetry breaking term in Ref. [30]. These results are plotted in the right panel of Fig. 2. The panel also presents an indirect estimation by NPLQCD Collaboration [43], which uses the result of $a_{0}$ in the $I=3 / 2 K \pi$ channel, since $a_{0}$ for $I=1 / 2$ and $I=3 / 2 K \pi$ channels share the common low energy constants in NLO ChPT formulae.

Lattice results are in rough accord with the phenomenological determination. While this is encouraging, in order to make more precise comparison with experiment, further direct calculations would be desired. In this conference, as shown in Fig. 2, RBC-UKQCD Collaboration [44] reports preliminary result of direct calculation of $a_{0}$ at the physical quark masses, where chiral extrapolation is not needed, in a large spatial extent of $5.5 \mathrm{fm}$. Furthermore, after the conference, Hadron Spectrum Collaboration presents the result at $m_{\pi}=390 \mathrm{MeV}$ [45].

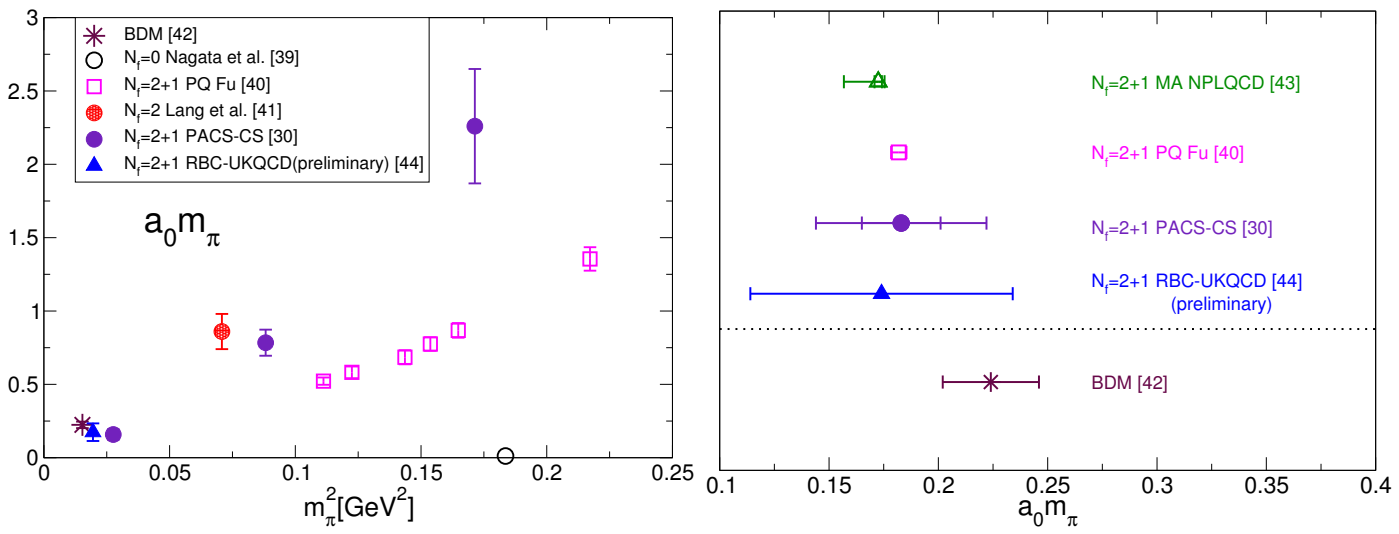

Figure 2: Left panel: $a_{0} m_{\pi}$ as a function of $m_{\pi}^{2}$ in the $I=1 / 2 K \pi$ channel. Right panel: $a_{0} m_{\pi}$ at physical $m_{\pi}$. Phenomenological determination by Büttiker et al. [42], and an indirect determination by NPLQCD Collaboration [43] are also shown. Burst symbol in left panel is shifted for clarify.

\section{Resonances}

It is possible to obtain the mass and decay width of resonances from the phase shift $\delta(p)$ of its final scattering state, at least in two-particle decay processes. For the $\rho$ meson decay into two pions, for example, the $\rho$ meson mass $m_{\rho}$ and an effective $\rho \pi \pi$ coupling $g_{\pi \pi \rho}$ can be extracted through the Breit-Wigner form,

$$
\frac{p^{3}}{\sqrt{s}} \cot \delta(p)=\frac{6 \pi}{g_{\pi \pi \rho}^{2}}\left(m_{\rho}^{2}-s\right),
$$


where $\sqrt{s}$ is the center-of-mass energy of the two pions. The decay width $\Gamma_{\rho}$ is then obtained by

$$
\Gamma_{\rho}=\frac{p_{\rho}^{3}}{m_{\rho}^{2}} \frac{g_{\rho \pi \pi}^{2}}{6 \pi}
$$

where $p_{\rho}$ is the relative momentum of the two pions at the resonance point $\left(s=m_{\rho}^{2}\right), p_{\rho}^{2}=m_{\rho}^{2} / 4-$ $m_{\pi}^{2}$. Therefore, when $\delta(p)$ is calculated by Lüscher's method or its extensions for at least two different values of $p, m_{\rho}$ and $g_{\pi \pi \rho}$ (or $\Gamma_{\rho}$ ) can be determined using eq.(4.1).

In order to obtain the phase shift for several different values of $p$, we might need to extract the excited state energy corresponding to higher scattering states. For this purpose, the variational method proposed by Lüscher and Wolff [46] is often used in lattice calculations.

\section{1 $I=1$ two-pion channel}

The decay process of the $\rho$ meson is an ideal resonance process for an initial study of scattering and decay, since the spectrum consists only of the $\rho$ meson and its final two-pion scattering state from the threshold $\left(2 m_{\pi}\right)$ to the vicinity of the resonance energy region. Computationally, however, this decay process is much harder than the $a_{0}$ calculation of the $I=2$ two-pion channel. One of the difficulties is the necessity of the rectangular diagram, as in the $I=1 / 2 K \pi$ channel, with a non-zero relative momentum of the two pions. Another difficulty is that, since the decay proceeds through the P-wave, the pion has to be sufficiently light and the spatial size $L$ sufficiently large so as to satisfy the decay condition,

$$
m_{\rho}^{2}>4\left(m_{\pi}^{2}+(2 \pi / L)^{2}\right)
$$

This condition can be relaxed if one uses the moving frame [3].

The first calculation of the $\rho$ meson decay was carried out in $N_{f}=2$ QCD by CP-PACS Collaboration [47] using the total momentum $|P|=1$ frame. After this work, five papers [48, $49,38,50,51]$ have been published. Figure 3 show $\delta(p)$ calculated with $m_{\pi}=390 \mathrm{MeV}$ by Hadron Spectrum Collaboration [51]. There are 29 data points obtained at different $p$ 's using several moving frames and irreducible representations on three different volumes. From the BreitWigner fit as in eq. (4.1), they extract the resonance mass $m_{R}=m_{\rho}$ and the coupling $g_{\pi \pi \rho}$ as shown in the figure.

Figure 4 presents a compilation of lattice results for $m_{\rho}$ (left panel) and $g_{\pi \pi \rho}$ (right panel) in Refs. [47, 48, 49, 38, 50, 51] extracted from the Breit-Wigner form fit. The experimental values are also plotted. For $m_{\rho}$ result, the $N_{f}=2$ results by ETM Collaboration are larger than the others. This discrepancy could be explained by a systematic error from scale setting. If we choose Sommer scale $r_{0}$ for the scale setting in all the results, discrepancy becomes smaller, for example, the results for ETM and PACS-CS Collaborations differ by less than 7\% [38].

The lattice results for $g_{\pi \pi \rho}$, presented in the right panel of Fig. 4 , show that it has only weak dependence on the pion mass, and that its value is consistent with experiment albeit errors are still generally large. We should note that the result by Hadron Spectrum Collaboration [51] with a small error does not quite agree with experiment, and so is that by Lang et al. [49] albeit with a better agreement. The discrepancies from the experiment are not decreased by choosing $r_{0}$ to set the scale. In this conference, two preliminary results by Fahy et al. [52] and BMW Collaboration [53] 
are reported as shown in Fig. 4. Fahy et al. [52] obtain the result at $m_{\pi}=240 \mathrm{MeV}$, and BMW Collaboration obtain the results in $m_{\pi}=134-300 \mathrm{MeV}$ [53].

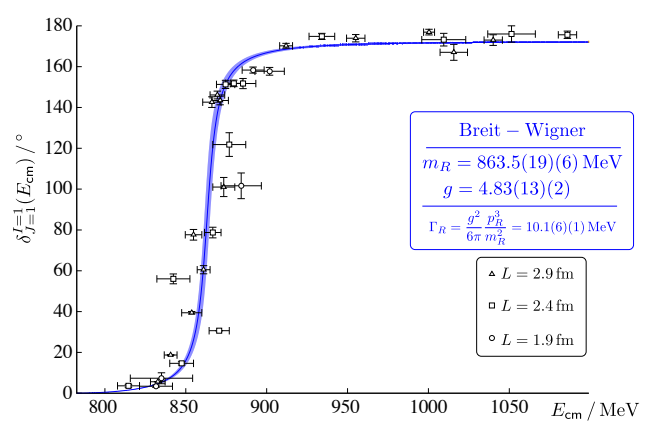

Figure 3: $I=1 \pi \pi$ phase shift calculated by Hadron Spectrum Collaboration [51].



Figure 4: Resonance mass $m_{\rho}$ (left panel) and the coupling $g_{\pi \pi \rho}$ (right panel) obtained in various calculations. Open circle is result with lattice dispersion relation [47].

\subsection{Other decay channels}

In the $\mathrm{P}$-wave $I=1 / 2$ channel, the $K^{*}$ meson decays to the $K \pi$ final state. This is an ideal decay process as in the $\rho$ meson decay; there are only $K \pi$ scattering and $K^{*}$ state in the energy region below the resonance. There have been three lattice calculations of $\delta(p)$ in this channel, by Fu and Fu [54] in $N_{f}=2+1$ QCD, Prelovsek et al. [55] in $N_{f}=2$ QCD, and Hadron Spectrum Collaboration [56, 45] in $N_{f}=2+1$ QCD. The last one is reported in this conference, and reviewed by other plenary speakers [13,14]. A difficulty of the lattice calculation in this channel is mixing of even and odd partial wave contributions in moving frame $[8,9,10,11,12]$ due to $m_{\pi} \neq m_{K}$. Some methods to solve the difficulty were proposed in Refs. [57, 55, 45].

In Ref. [54] it is assumed that S-wave contribution is negligible, so that the result contains systematic errors from the mixing. Prelovsek et al. [55] obtain a value consistent with the experiment, $g_{\pi K K^{*}}=5.7(1.6)$, at $m_{\pi}=270 \mathrm{MeV}$. Hadron Spectrum Collaboration [56, 45] calculate $\delta(p)$ at 
$m_{\pi}=390 \mathrm{MeV}$, while the $K^{*}$ meson is a bound state at this value of $m_{\pi}$. Hadron Spectrum Collaboration also carry out coupled channel analyses in S- and D-wave channels to extract $\delta(p)$ for each of $\pi K$ and $\eta K$ scatterings and inelasticity.

We should add that studies related to the decay channels, $N^{*} \rightarrow N \pi[58], \sigma \rightarrow \pi \pi[59,60]$, and $a_{0} \rightarrow \pi \eta[61]$ are reported at this conference.

\section{Comparisons of Lüscher's and HALQCD methods}

In this section, recent results obtained by Lüscher's method and HALQCD method are compared in three channels, $I=2$ two-pion channel, H-dibaryon $(\Lambda \Lambda)$ channel, and two-nucleon $N N$ channel. After the comparison, possible uncertainties in each of the two methods are discussed.

\subsection{HALQCD method}

The HALQCD method [62, 63] extracts the potential between two particles, $V(\vec{r})$, from NambuBethe-Salpeter (NBS) wave function. The idea derives from the derivation of Lüscher's formula in quantum mechanics [2] and in quantum field theory [64], and also from calculations of the NBS wave function in two-dimensional scalar field theory [65] and $I=2$ two-pion channel [66]. While these studies concentrated on the behavior of NBS wave function at large $r$, where $V(\vec{r}) \approx 0$, the HALQCD method asserts that dynamics could be extracted from the smaller $r$ region.

The NBS wave function of the $n$-th $N N$ state, $\phi_{n}(\vec{r})$, in $N N$ channel is calculated from fourpoint function of $N$ operator,

$$
C_{N N}(\vec{r}, t)=\sum_{\vec{x}}\langle 0|N(\vec{x}+\vec{r}, t) N(\vec{x}, t) \overline{N N}(0)| 0\rangle=\sum_{n} A_{n} \phi_{n}(\vec{r}) e^{-W_{n} t}+\cdots,
$$

where $\overline{N N}$ is a $N N$ source operator, $W_{n}$ is the $n$-th energy, $W_{n}=2 \sqrt{m_{N}^{2}+p_{n}^{2}}, A_{n}=\left\langle N N, W_{n}|\overline{N N}| 0\right\rangle$, and

$$
\phi_{n}(\vec{r})=\sum_{\vec{x}}\left\langle 0|N(\vec{x}+\vec{r}) N(\vec{x})| N N, W_{n}\right\rangle
$$

The spin index of the $N$ operator is omitted for simplicity. The dots represent contributions from inelastic scattering states. A non-local potential $U\left(\vec{r}, \vec{r}^{\prime}\right)$ is defined by acting with the Laplacian on $\phi_{n}(\vec{r})$,

$$
\left(\frac{\nabla^{2}}{m_{N}}+\frac{p_{n}^{2}}{m_{N}}\right) \phi_{n}(\vec{r})=\int d^{3} r^{\prime} U\left(\vec{r}, \vec{r}^{\prime}\right) \phi_{n}\left(\vec{r}^{\prime}\right)
$$

Assuming that the higher order terms of the velocity expansion of $U\left(\vec{r}, \vec{r}^{\prime}\right)$ are negligible,

$$
U\left(\vec{r}, \vec{r}^{\prime}\right) \approx V(\vec{r}) \delta\left(\vec{r}-\vec{r}^{\prime}\right)
$$

a local potential $V(\vec{r})$ is obtained from eq.(5.3). It should be noted that in quantum field theory the right-hand side of eq.(5.3) corresponds to Fourier transformation of off-shell scattering amplitude [66], which is related to $\delta(p)$ only on shell.

One way to calculate $V(\vec{r})$ is to use the ground state NBS wave function $\phi_{0}(\vec{r})$ extracted from $C_{N N}(\vec{r}, t)$ for large $t$. In the $N N$ case, however, statistical noises increase exponentially at large $t$, 
rendering an accurate calculation of $C_{N N}(\vec{r}, t)$ difficult. It was proposed in Ref. [67] to employ the time dependence of $C_{N N}(\vec{r}, t)$ and rewrite eq. (5.3) as,

$$
\left(\frac{\nabla^{2}}{m_{N}}+\frac{1}{4 m_{N}} \frac{\partial^{2}}{\partial t^{2}}-\frac{\partial}{\partial t}\right) \bar{C}_{N N}(\vec{r}, t)=V(\vec{r}) \bar{C}_{N N}(\vec{r}, t),
$$

where $\bar{C}_{N N}(\vec{r}, t)=C_{N N}(\vec{r}, t) \exp \left(2 m_{N} t\right)$. A key assumption for this equation is that all $\phi_{n}(\vec{r})$ 's in $C_{N N}(\vec{r}, t)$ yield a common $V(\vec{r})$, in other words, eq.(5.4) is valid. With eq.(5.5), $V(\vec{r})$ can be obtained from $C_{N N}(\vec{r}, t)$ at smaller $t$ region as long as contributions of inelastic states are negligible.

In the HALQCD method, thus, the potential $V(\vec{r})$ obtained is fitted by a continuous function of $r$, like the Yukawa potential plus some hard core, which is then employed to calculate the phase shift $\delta(p)$ by solving the Schrödinger equation. An assumption in calculating $\delta(p)$ is that finite volume effects are negligible in $V(\vec{r})$. It is also assumed that, while the potential $V(\vec{r})$ may depend on the choice of the sink operator employed for $C_{N N}(\vec{r}, t)$ in eq.(5.1), such a dependence does not affect the phase shift $\delta(p)$ evaluated from the potential.

In the left panel of Fig. 5 we present a recent result of HALQCD for the nuclear potential of the spin-singlet ${ }^{1} \mathrm{~S}_{0}$ channel calculated in $N_{f}=2+1$ QCD at $m_{\pi}=410-700 \mathrm{MeV}$ [68]. The potential has the features similar to the phenomenological nuclear potentials with a repulsive core in the smaller $r$ region and a one-pion exchange potential at large $r$. The depth of the lattice potential seems shallower than the phenomenological ones, which is considered to be caused by a heavy $m_{\pi}$ used in the calculation. The right panel of Fig. 5 presents the results of $\delta(p)$ at each $m_{\pi}$ calculated from $V(r)$.

In this conference, using the HALQCD method, an update of the $\Omega \Omega$ potential [69] and a study of the quark mass dependence of the three-nucleon potential [70] are presented by HALQCD Collaboration.
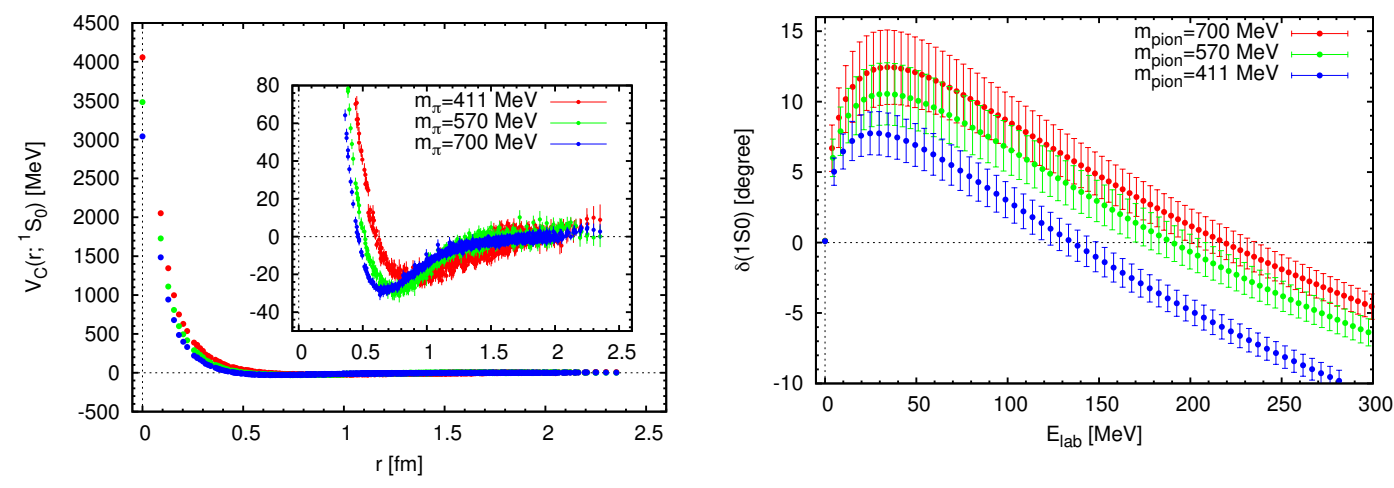

Figure 5: Recent result of nuclear potential (left panel) and $\delta(p)$ obtained from the potential (right panel) in $N_{f}=2+1$ QCD calculated by HALQCD Collaboration [68].

\section{$5.2 I=2$ two-pion scattering}

Kurth et al. [71] calculate $\delta(p)$ for the $I=2$ two-pion channel by the two methods for $N_{f}=0$ QCD at $m_{\pi}=940 \mathrm{MeV}$ on four volumes from $L=1.8$ to $5.5 \mathrm{fm}$. The stability of $V(r)$ calculated by 
the $t$-dependent method of eq.(5.5) is illustrated in the left panel of Fig. 6, where different colors correspond to different $t$ ranges, $t=15-48\left(R_{1}\right), 24-48\left(R_{2}\right)$, and 33-48( $\left.R_{3}\right)$. The right panel of Fig. 6 compares $\delta(p)$ calculated from $V(r)$ (solid lines) with that extracted by Lüscher's method (filled symbols). For this repulsive channel with a relatively simple shape of $V(r)$, we observe an agreement of the two methods for small momenta.
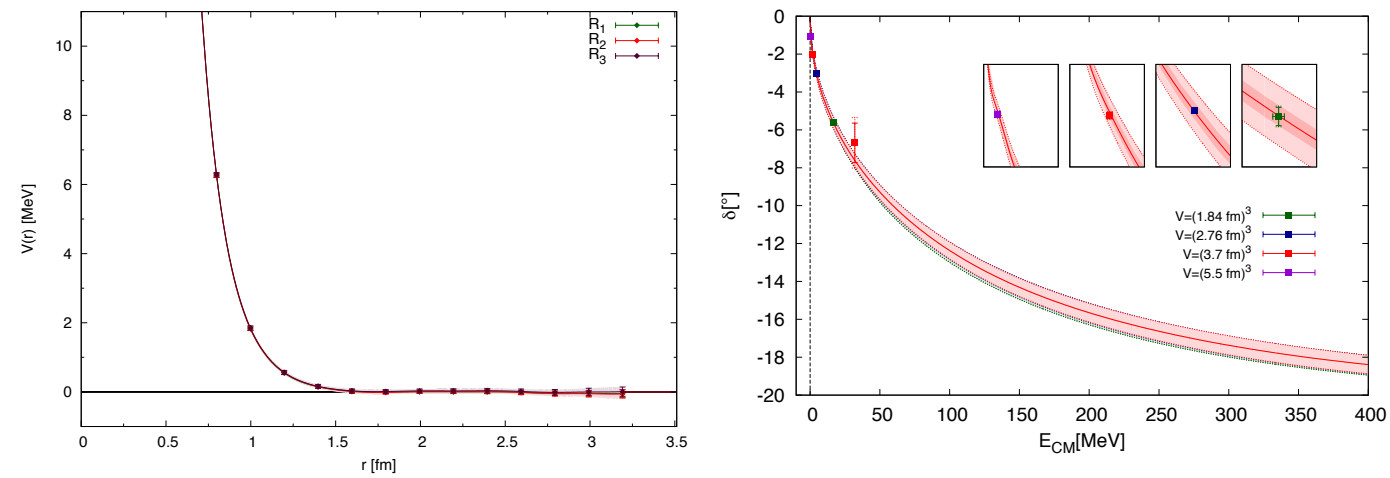

Figure 6: Potential in $I=2 \pi \pi$ channel (left panel) and $\delta(p)$ obtained from the potential (right panel) in quenched QCD calculated by Kurth et al. [71].

\section{$5.3 H$-dibaryon channel}

HALQCD Collaboration [72, 73] examines the formation of H-dibaryon as a bound state in the $\Lambda \Lambda$ channel in $N_{f}=3$ QCD. The result of $V(r)$ at $m_{\pi}$ in the range $470-1200 \mathrm{MeV}$ obtained by the HALQCD method is shown in the left panel of Fig. 7, which exhibits an attractive core in contrast to the $N N$ case. Fitting $V(r)$ and solving the Schrödinger equation, a bound state is found for all values of $m_{\pi}$ examined.

The right panel of Fig. 7 shows the result from Lüscher's method for $N_{f}=3$ at $m_{\pi}=800 \mathrm{MeV}$ by NPLQCD Collaboration [20]. The effective energy shift of the ground $\Lambda \Lambda$ state from $2 m_{\Lambda}$ is clearly non-zero in the plateau region. After investigating the volume dependence of the energy shift, it is concluded that the ground state is a bound state.

The binding energies calculated in the two methods are plotted in the left panel of Fig. 8 where we also added the result of NPLQCD Collaboration for $N_{f}=2+1$ [17]. Qualitatively, the two methods are in agreement in that both predict bound states for the range of $m_{\pi}$ examined. In quantitative detail, they differ significantly at $m_{\pi} \sim 800 \mathrm{MeV}$.

In this conference, Green et al. report a preliminary result [74] in $N_{f}=2$ QCD at $m_{\pi}=1 \mathrm{GeV}$ and $450 \mathrm{MeV}$. They employ the variational method [46] with local products of six quark operator for both source and sink time slices, and conclude that the ground state has higher energy than $2 m_{\Lambda}$, as presented in the right panel of Fig. 8 for the case of $m_{\pi}=450 \mathrm{MeV}$, in contrast to NPLQCD result. A difference between NPLQCD's and their calculations is sink operator. NPLQCD Collaboration uses two-baryon sink operator. After the conference, a preliminary result of calculation with a two-baryon operator is presented in other conference [75], and they observe smaller ground state energy than $2 m_{\Lambda}$. 

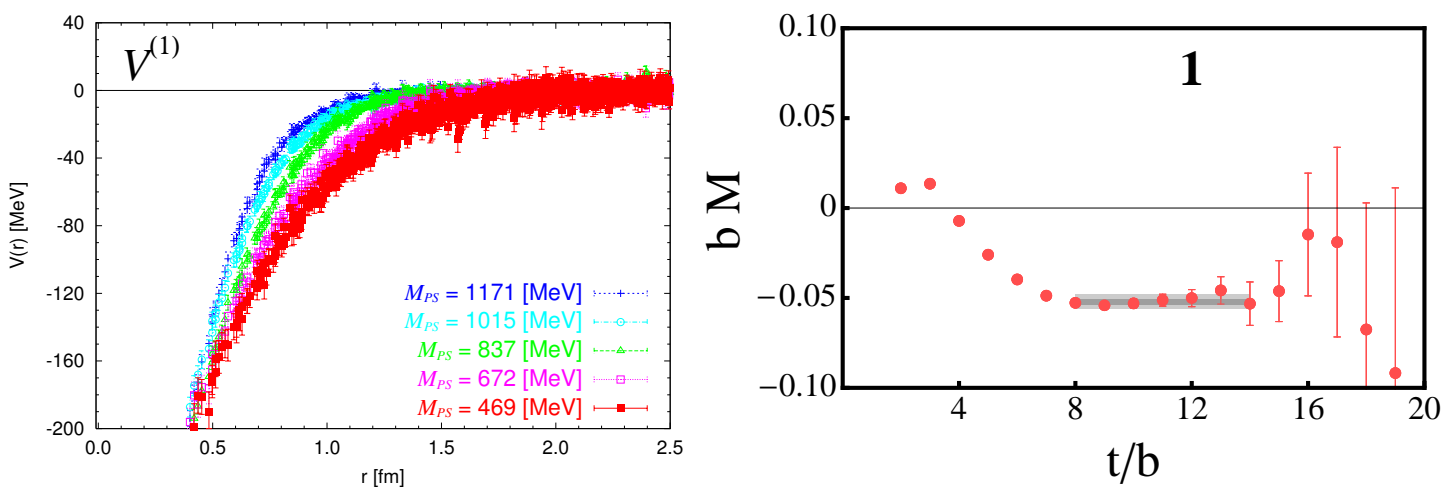

Figure 7: Potential calculated by HALQCD Collaboration [73] (left panel) and effective energy shift calculated by NPLQCD Collaboration [20] (right panel) in $N_{f}=3 \mathrm{H}$-dibaryon channel.
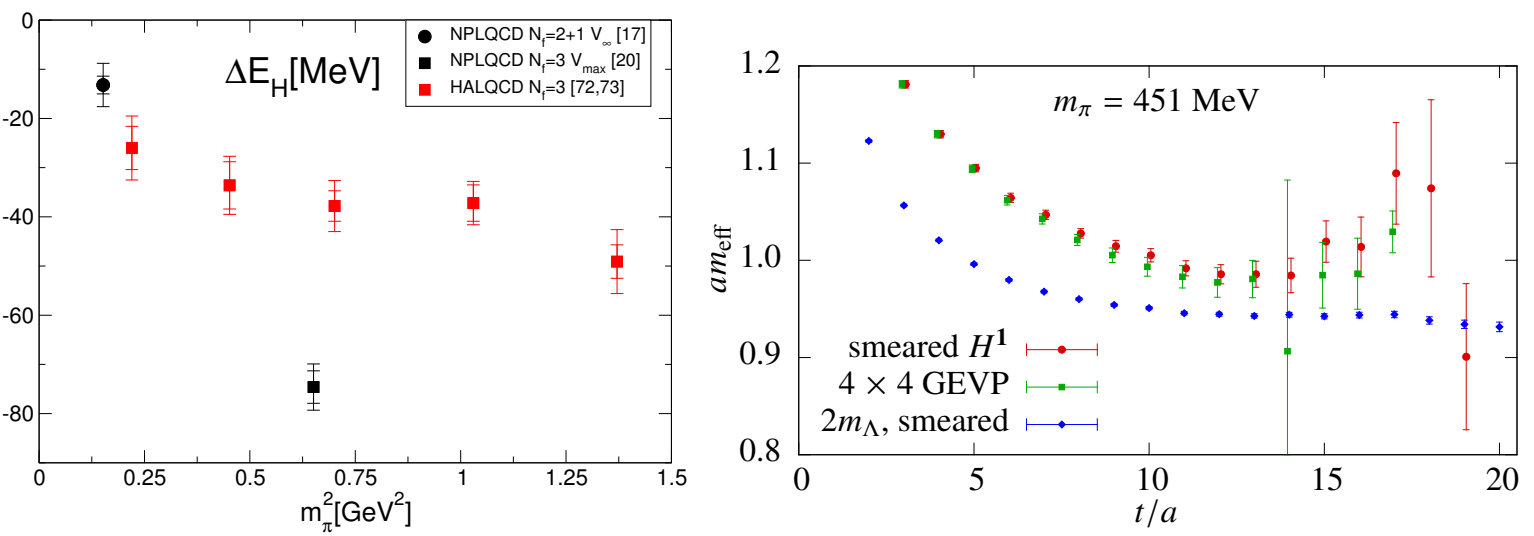

Figure 8: Binding energy obtained from Lüscher's and HALQCD methods (left panel) and preliminary result of effective energy shift calculated by Green et al. [74] (right panel) in H-dibaryon channel. $\mathrm{V}_{\infty}$ and $\mathrm{V}_{\max }$ in the left panel express results in the infinite volume and on the largest volume, respectively.

\subsection{Two-nucleon channels}

Two nucleons can be in either in the spin-triplet ${ }^{3} \mathrm{~S}_{1}$ channel or in the spin-singlet ${ }^{1} \mathrm{~S}_{0}$; in nature the former has a deuteron, while there are no bound states in the latter.

Figure 9 summarizes all results obtained so far by Lüscher's method [76, 77, 18, 78, 20, 19, 21] for the energy shift of the ground $N N$ state from $2 m_{N}$ in the spin-triplet ${ }^{3} \mathrm{~S}_{1}$ (left panel) and spinsinglet ${ }^{1} \mathrm{~S}_{0}$ (right panel) channels. Early studies $[76,77]$ did not examine the volume dependence of the energy shift, so that it is not clear if the ground state is a scattering state or a bound state. Recent studies consciously examine this issue by working with multiple volumes [18, 78, 20, 19, 21]. Except for one result [78] (up triangle) which unfortunately suffers from large error, all results obtained by study of volume dependence have found that the ground states in both the channels are bound states. The existence of those bound states at the large $m_{\pi}$ is also confirmed by the sign of scattering length $a_{0}$ in each channel $[18,20]$ based on the discussion in Refs. [15, 16].

In the spin-triplet ${ }^{3} \mathrm{~S}_{1}$ channel, the bound state nature found in lattice calculations is consistent with the presence of deuteron in nature. The magnitude of binding energy, however, is significantly larger, and does not show a trend of decrease toward the experimental value as $m_{\pi}$ is taken lighter, 
at least to $m_{\pi}=300 \mathrm{MeV}$ [21]. The trend is similar in the spin-singlet ${ }^{1} \mathrm{~S}_{0}$ channel. There is a bound state down to $m_{\pi}=300 \mathrm{MeV}$, and it is not clear if or how this bound state disappears as one approaches the physical pion mass.

A recent result for $\delta(p)$ in the ${ }^{1} \mathrm{~S}_{0}$ channel obtained by the HALQCD method [68] is shown in the right panel of Fig 5. If there is a bound state, $\delta(p)$ should start at 180 degrees at $p=0$. The figure therefore shows that there is no bound state in this channel at $m_{\pi}=410-700 \mathrm{MeV}$. In contrast to the results from Lüscher's method described above, this is consistent with experiment. Quantitatively, however, the magnitude of phase shift in experiment is much larger than the values in Fig. 5. It would be important to perform a calculation at the physical $m_{\pi}$ to reproduce the experimental result. Bound state is also not observed in the ${ }^{3} \mathrm{~S}_{1}$ channel by the HALQCD method [73]. The $\delta(p)$, though increasing with decreasing $m_{\pi}$, does not support a bound state down to $m_{\pi} \approx 470 \mathrm{MeV}$ in $N_{f}=3$ QCD. This situation is opposite to that with the Lüscher's method.

We have to conclude that at present the results from the two methods in the $N N$ channels do not agree, even for the presence or absence of bound states.
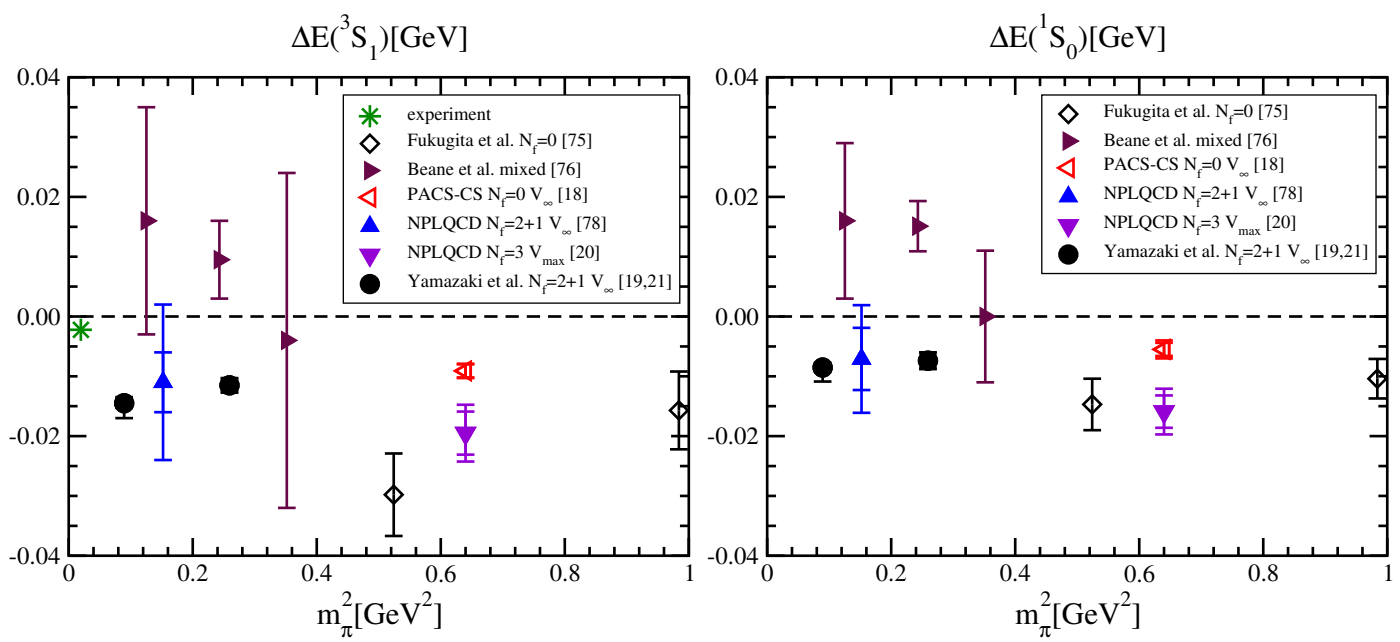

Figure 9: Energy shift in $N N{ }^{3} \mathrm{~S}_{1}$ (left) and ${ }^{1} \mathrm{~S}_{0}$ (right) channels. Experimental value of deuteron is also plotted in left panel. $V_{\infty}$ and $V_{\max }$ express results in the infinite volume and on the largest volume, respectively.

\subsection{Systematic uncertainty}

We have seen that the results from the two methods agree with each other at least qualitatively for the $I=2$ two-pion and $H$-dibaryon channels, while they differ in an essential way for the $N N$ channels. A possible reason for this difference may be that the first two cases are simple two-body systems governed by potentials which are monotone functions of $r: V(r)$ in the $I=2$ two-pion channel decreases as $r$ increases (Fig. 6), and the one in the $H$-dibaryon channel increases as $r$ (Fig. 8). The $N N$ potential, however, has a more complex structure (Fig. 5) with a repulsive core near the origin and an attractive tail at large distances. Thus, various systematic uncertainties might affect a balance toward bound state formation in a serious way. Let us then discuss possible systematic uncertainties in the two methods for the $N N$ channels. 


\subsubsection{Lüscher's method}

For bound states, Lüscher's method is nothing but the traditional calculation of evaluating the mass of the ground state of hadrons from two-point correlation function, and extrapolating it to the infinite volume. Thus, the crucial point we need to worry about is how reliably the ground state energy is extracted for a given volume. Figure 10 presents the effective energy shift for the $N N$ state from $2 m_{N}$ in the ${ }^{3} \mathrm{~S}_{1}$ channel by Yamazaki et al. [19] and NPLQCD Collaboration [20]. While a clear plateau extends only over 3 or 4 time slices, the negative value of the energy shift appears quite convincing.

One should note that the results above are obtained with only one type of source operator. It is a legitimate question to ask if one obtains the same result, when a different source operator is employed. Similarly, the dependence on the choice of the sink operator is also a potential issue. These issues have not been thoroughly investigated. Application of the variational method [46] would be worthwhile to settle these issues.

There are two more sources of possible errors which, however, are more dynamical in nature. One is the dependence on the quark mass. It may well be that the bound state found in the ${ }^{1} \mathrm{~S}_{0}$ channel for the pion mass down to $m_{\pi} \approx 300 \mathrm{MeV}$ [21] disappears at a smaller pion mass but heavier than the physical mass.

Another is the dependence on the lattice spacing. It is possible in principle that the pion mass $m_{\pi}^{c}$ below which a bound state in the spin-singlet ${ }^{1} \mathrm{~S}_{0}$ channel disappears varies with the lattice spacing. It may require a sufficiently small lattice spacing before $m_{\pi}^{c}$ becomes non-zero or larger than the physical value.

These possibilities can only be settled by repeating the calculations toward the physical pion mass and for smaller lattice spacings.
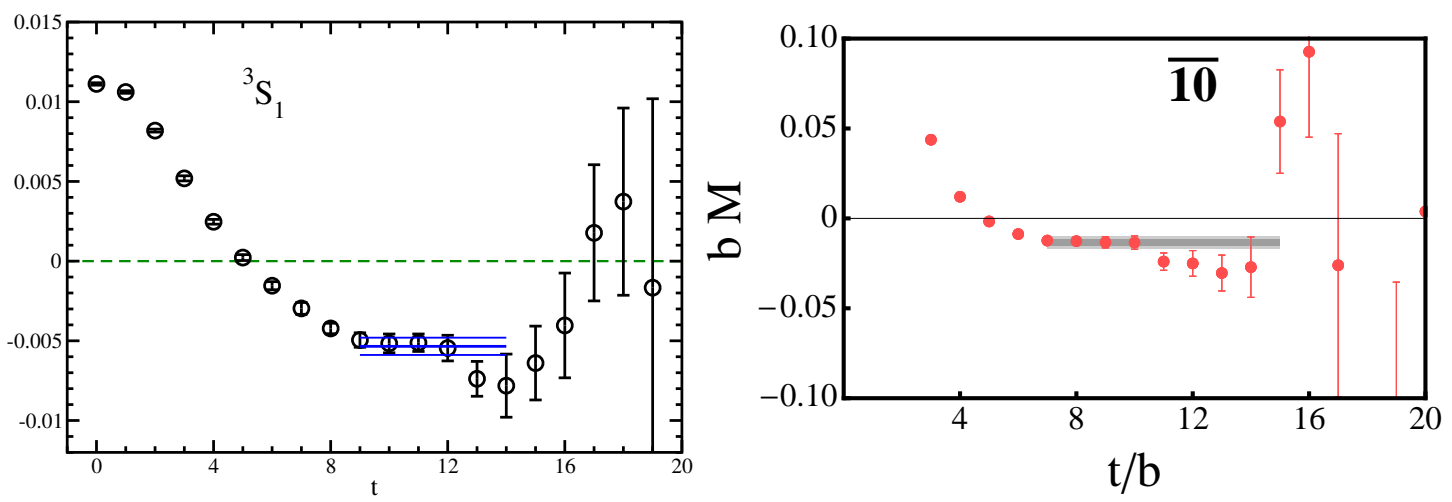

Figure 10: Effective energy shift calculated by Yamazaki et al. [19] (left) and NPLQCD Collaboration [20] (right) in $N N^{3} \mathrm{~S}_{1}$ channel.

\subsubsection{HALQCD method}

The HALQCD method relies crucially on the potential $V(\vec{r})$ extracted from the NBS wave function. Hence possible systematic errors center around the meaning of the potential and how the final physics results may or may not depend on the ambiguities in the potential. Let us discuss possible sources of systematic errors and ambiguities one by one. 
(i) Sink operator dependence of $V(\vec{r})$ and $\delta(p)$

The potential $V(\vec{r})$ will in general depend on the choice of the sink operator of $C_{N N}(\vec{r}, t)$ in eq.(5.1). In SU(2) gauge theory, Takahashi et al. [79] examined this issue, and found that $V(\vec{r})$ in the small $r$ region largely depends on the choice of the sink operator, while the dependence is small for large $r$. To what extent such a dependence affects the phase shift extracted from $V(\vec{r})$ was not tested in this study, but this is certainly a point which requires a detailed check.

(ii) Velocity expansion of $U\left(\vec{r}, \vec{r}^{\prime}\right)$

If higher order of velocity expansion is not negligible in eq.(5.4), $V(\vec{r})$ obtained from eq.(5.3) or (5.5) depends on the relative momentum $p$ of the two-nucleon state. The dependency was investigated by Murano et al. [80] at $p \sim 0$ and $250 \mathrm{MeV}$, and it was concluded that the $V(\vec{r})$ obtained from two values of $p$ agree within the statistical error.

(iii) Volume dependence of $V(\vec{r})$

If the extracted potential $V(\vec{r})$ has finite volume effects, it may affect $\delta(p)$ and hence the presence or absence of bound states. Thus, finite volume dependence was investigated by HALQCD Collaboration [72] at $m_{\pi}=1 \mathrm{GeV}$ in the spatial extent of 2-3 fm, and it was concluded that the finite volume effects in $V(\vec{r})$ is negligible within the statistical error.

(iv) Lattice spacing dependence of $V(\vec{r})$

Lattice spacing dependence of $V(\vec{r})$ was examined at $m_{\pi}=1.1 \mathrm{GeV}$ for three different lattice spacings, $a=0.11-0.22 \mathrm{fm}$, by HALQCD Collaboration [81]. They found that lattice spacing dependence is small in the larger $r$ region, but it is large in the small $r$ region, $r \lesssim 0.5 \mathrm{fm}$, and then $\delta(p)$ 's obtained at different lattice spacings differ at large $p$ region.

(v) Determination of $m_{N}$

If the value of $m_{N}$ contains systematic errors, which is even a few $\mathrm{MeV}$, it corresponds to a constant shift of $V(r)$ for all values of $r$, since

$$
V(\vec{r}) \approx \frac{\left(\frac{\nabla^{2}}{m_{N}}-\frac{\partial}{\partial t}\right) C_{N N}(\vec{r}, t)}{C_{N N}(\vec{r}, t)}-2 m_{N} .
$$

The tiny constant shift of $V(r)$ might be crucial in some case, e.g., identification of existence of bound state.

(vi) $V(\vec{r})$ in large $r$ region

It is hard to extract $V(\vec{r})$ in the large $r$ region precisely, because the noise-to-signal ratio of $V(\vec{r})$ increases with $r$. Since the large $r$ region is important to determine how long attractive tail of the potential continues, this uncertainty might affect existence of bound state as pointed out by the plenary speaker in the last conference [82].

To sum up the considerations above, most uncertain with the HALQCD method seems to be the ambiguities in the potential for small values of $r$ as written in (i), (iii), and (iv). The small $r$ region is not relevant to calculate $\delta(p)$ at small $p$, while it becomes important to calculate $\delta(p)$ at large $p$, and also to test the existence of a bound state. Thus, those uncertainties in the small $r$ region might cause the difference of the result obtained from the two methods.

\section{Light nuclei}

A first attempt to simulate the formation of helium nuclei in lattice QCD was reported in 
Ref. [83]. In this work, the binding energies of the ${ }^{4} \mathrm{He}$ and ${ }^{3} \mathrm{He}$ nuclei were calculated in quenched QCD at $m_{\pi}=800 \mathrm{MeV}$, by examining the volume dependence of the energy shift. A serious computational problem with nuclei calculations is huge number of Wick contraction. For helium nuclei [83], this problem was overcome by omitting calculations of redundant contractions under symmetries of interpolating operator, and by utilizing blocks of three quark propagators. Recently, more efficient calculation methods $[84,85,86]$ have been proposed. In this conference a preliminary result in this direction [87] is reported.

The exploratory study of helium nuclei has been followed by calculations in $N_{f}=3$ QCD at $m_{\pi}=810 \mathrm{MeV}$ [20], and 2+1 QCD at $m_{\pi}=510 \mathrm{MeV}$ [19] and $m_{\pi}=300 \mathrm{MeV}$ [21]. Results for the binding energies for ${ }^{4} \mathrm{He}$ and ${ }^{3} \mathrm{He}$ nuclei are summarized in Fig. 11. For both the nuclei, the quenched and $N_{f}=3$ calculations at $m_{\pi} \sim 800 \mathrm{MeV}$ give different results. While it might be a dynamical quark effect, precise understanding is lacking at present. At $m_{\pi}=300$, the lightest pion explored so far, the binding energy of ${ }^{4} \mathrm{He}$ is roughly consistent with the experiment, while that for ${ }^{3} \mathrm{He}$ is about three times larger than the experiment. The discrepancy between the lattice and experimental results might be caused by a large $m_{\pi}$ in the calculation. Future calculations closer to the physical $m_{\pi}$ should tell if this expectation is justified.

In the right panel of Fig. 11 the result from HALQCD method for ${ }^{4} \mathrm{He}$ in $N_{f}=3$ QCD [73], giving a very small binding energy, is also plotted. Results for ${ }^{3} \mathrm{He}$ is not available from the HALQCD method.
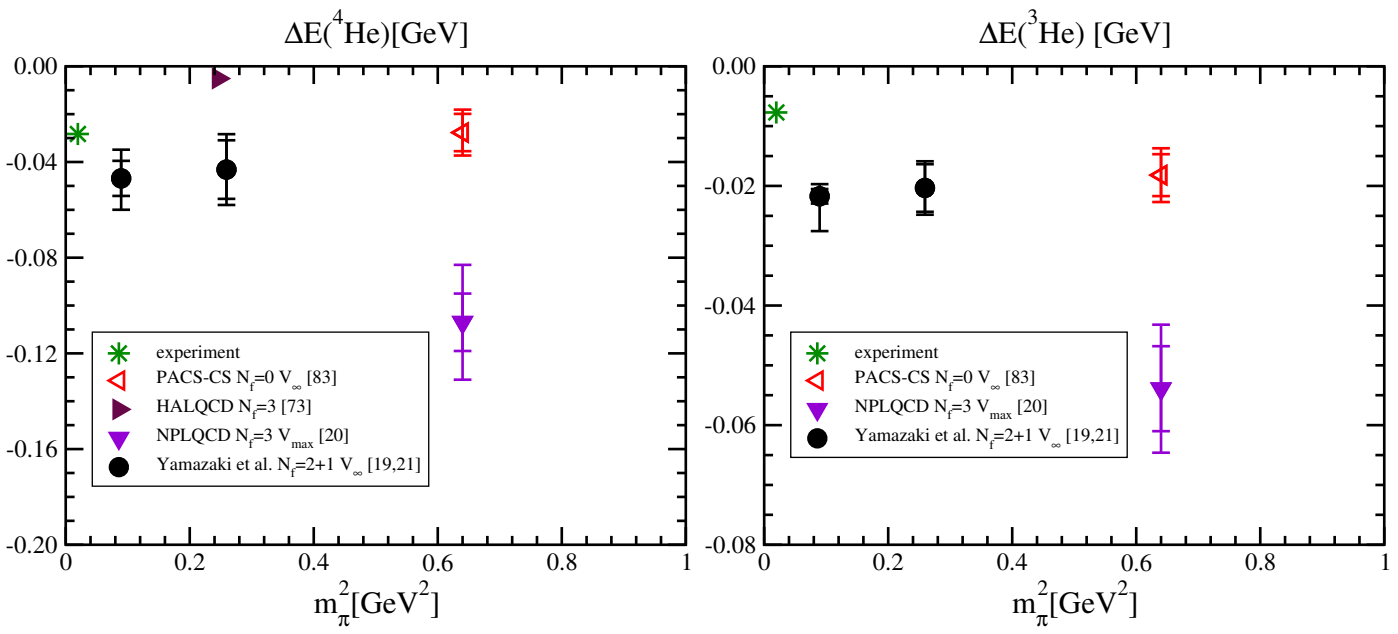

Figure 11: Binding energies for ${ }^{4} \mathrm{He}$ (left) and ${ }^{3} \mathrm{He}$ (right) channels. Experimental values are also plotted. $\mathrm{V}_{\infty}$ and $\mathrm{V}_{\max }$ express results in the infinite volume and on the largest volume, respectively.

Let us list some related studies for nuclei. NPLQCD Collaboration calculates magnetic moments for light nuclei [88] and binding energies for quarkonium-nucleus bound states [89]. HALQCD Collaboration calculates the binding energy of spin-2 $N \Omega$ bound state [90] and medium-heavy nuclei [73] from potentials obtained by the HALQCD method. The nuclei calculation has been extended to theories beyond the standard model. Detmold et al. [91] calculate nuclei of two to four particles in two-flavor SU(2) gauge theory to explore the importance of nuclear physics in the strongly-interacting dark matter models. 


\section{Conclusion}

In this report we review recent works for scattering and decay channels. The scattering length in $I=2$ two-pion channel is precisely determined using chiral extrapolation with ChPT formula. Still careful estimation of systematic errors are necessary, some recent results have smaller error than the experimental results. Thus, the calculation in this channel enters precision measurement era as in hadron masses and decay constants. The calculation of scattering length in $I=1 / 2 K \pi$ channel is more difficult than the one in $I=2$ two-pion channel, so that only a few calculations are carried out. The resonance mass and decay width are extracted from scattering phase shift. In $I=1$ two-pion channel, the $\rho$ meson mass and its coupling to two pions are calculated. Both the physical quantities are roughly consistent with the experiment within large error of lattice results.

Comparisons of the results from Lüscher's method and HALQCD method are discussed. In $I=2$ two-pion channel, the results from the two methods agree with each other numerically. While the binding energies of $H$-dibaryon differ in larger $m_{\pi}$ region, the two method give the consistent conclusion that the $H$-dibaryon exists in larger $m_{\pi}$ than physical one. However, in the $N N$ channels, the two methods obtain opposite results in the point of view of the presence of bound states. Possible uncertainties in each method are discussed in the $N N$ channels. Contaminations from higher excited states, larger quark mass, and finite lattice spacing could affect the result from Lüscher's method. For HALQCD method, several possible uncertainties are listed. Uncertainties of the potential in small $r$ region might explain why the bound state is not formed in this method. In order to understand the difference between the results obtained from Lüscher's method and HALQCD method, it would be necessary to carry out more detail investigations for uncertainties in both the methods. Furthermore, understanding of relation between the potential evaluated from NBS wave function, in other words, Fourier transformation of off-shell scattering amplitude, and phase shift in quantum field theory might shed light on the difference.

The helium nuclei are calculated in several works using Lüscher's method. In the ${ }^{4} \mathrm{He}$ channel the binding energy is roughly consistent with the experiment, while in the ${ }^{3} \mathrm{He}$ channel it is larger than the experiment. The discrepancy of the binding energy from the experiment is considered to be mainly caused by large $m_{\pi}$ in the calculation. Therefore, calculations at smaller $m_{\pi}<300$ $\mathrm{MeV}$ would be necessary to study whether lattice calculation reproduces the experimental binding energy or not.

\section{Acknowledgements}

I would like to thank John Bulava, Raul Briceño, William Detmold, Jeremy Green, Yoshinobu Kuramashi, Christian Lang, Thibaut Metivet, Colin Morningstar, Hidekatsu Nemura, Sasa Prelovsek, and Akira Ukawa for discussions and sending comments and figures for this talk. This work is partially supported by the JSPS Grant-in-Aid for Scientific Research for Young Scientists (B) No.25800138, and also by Grants-in-Aid of the Japanese Ministry for Scientific Research on Innovative Areas No.23105708.

\section{References}

[1] M. Lüscher, Commun. Math. Phys. 105 (1986) 153-188. 
[2] M. Lüscher, Nucl. Phys. B354 (1991) 531-578.

[3] K. Rummukainen and S. A. Gottlieb, Nucl.Phys. B450 (1995) 397-436, [hep-lat/ 9503028 ].

[4] C. Kim, C. Sachrajda, and S. R. Sharpe, Nucl.Phys. B727 (2005) 218-243, [hep-lat/ 0507006 ].

[5] N. H. Christ, C. Kim, and T. Yamazaki, Phys.Rev. D72 (2005) 114506, [hep-lat/ 0507009 ].

[6] ETM Collaboration, X. Feng, K. Jansen, and D. B. Renner, PoS LATTICE2010 (2010) 104, [arXiv:1104.0058].

[7] J. J. Dudek, R. G. Edwards, and C. E. Thomas, Phys.Rev. D86 (2012) 034031, [arXiv:1203.6041].

[8] Z. Fu, Phys.Rev. D85 (2012) 014506, [arXiv:1110.0319].

[9] L. Leskovec and S. Prelovsek, Phys.Rev. D85 (2012) 114507, [arXiv: 1202 . 2145].

[10] M. Doring, U. Meissner, E. Oset, and A. Rusetsky, Eur.Phys.J. A48 (2012) 114, [arXiv:1205.4838].

[11] M. Gockeler, R. Horsley, M. Lage, U.-G. Meissner, P. Rakow, et. al., Phys.Rev. D86 (2012) 094513, [arXiv:1206.4141].

[12] N. Li and C. Liu, Phys.Rev. D87 (2013) 014502, [arXiv:1209.2201].

[13] R. A. Briceño, arXiv:1411.6944.

[14] S. Prelovsek, PoS LATTICE2014 (2014) 015, [arXiv: 1411.0405 ].

[15] S. R. Beane, P. F. Bedaque, A. Parreno, and M. J. Savage, Phys. Lett. B585 (2004) 106-114, [hep-lat/0312004].

[16] S. Sasaki and T. Yamazaki, Phys. Rev. D74 (2006) 114507, [hep-lat/ 0610081$].$

[17] NPLQCD Collaboration, S. Beane et. al., Phys.Rev.Lett. 106 (2011) 162001, [arXiv:1012.3812].

[18] PACS-CS Collaboration, T. Yamazaki, Y. Kuramashi, and A. Ukawa, Phys. Rev. D84 (2011) 054506, [arXiv:1105.1418].

[19] T. Yamazaki, K.-i. Ishikawa, Y. Kuramashi, and A. Ukawa, Phys.Rev. D86 (2012) 074514, [arXiv: 1207.4277$]$.

[20] S. Beane, E. Chang, S. Cohen, W. Detmold, H. Lin, et. al., Phys.Rev. D87 (2013), no. 3 034506, [arXiv:1206.5219].

[21] T. Yamazaki, K.-i. Ishikawa, Y. Kuramashi, and A. Ukawa, arXiv:1502.0418.

[22] CP-PACS Collaboration, T. Yamazaki et. al., Phys. Rev. D70 (2004) 074513, [hep-lat/0402025].

[23] NPLQCD Collaboration, S. R. Beane, P. F. Bedaque, K. Orginos, and M. J. Savage, Phys.Rev. D73 (2006) 054503, [hep-lat/0506013].

[24] NPLQCD Collaboration, S. R. Beane, T. C. Luu, K. Orginos, A. Parreno, M. J. Savage, et. al., Phys.Rev. D77 (2008) 014505, [arXiv:0 006.3026$].$

[25] RBC-UKQCD Collaboration, Q. Liu, PoS LAT2009 (2009) 101, [arXiv: 0910 . 2658 ].

[26] X. Feng, K. Jansen, and D. B. Renner, Phys.Lett. B684 (2010) 268-274, [arXiv: 0909.3255$].$ 
[27] T. Yagi, S. Hashimoto, O. Morimatsu, and M. Ohtani, arXiv:1108.2970.

[28] Z. Fu, Commun.Theor.Phys. 57 (2012) 78-84, [arXiv:1110.3918].

[29] Z. Fu, Phys.Rev. D87 (2013) 074501, [arXiv:1303.0517].

[30] PACS-CS Collaboration, K. Sasaki, N. Ishizuka, M. Oka, and T. Yamazaki, Phys.Rev. D89 (2014) 054502, [arXiv:1311.7226].

[31] S. Pislak, R. Appel, G. Atoyan, B. Bassalleck, D. Bergman, et. al., Phys.Rev. D67 (2003) 072004, [hep-ex/ 030104 0]. [Erratum-ibid. D81 (2010) 119903].

[32] NA48-2 Collaboration, J. Batley et. al., Eur.Phys.J. C70 (2010) 635-657.

[33] Hadron Spectrum Collaboration, J. J. Dudek, R. G. Edwards, M. J. Peardon, D. G. Richards, and C. E. Thomas, Phys.Rev. D83 (2011) 071504, [arXiv:1011.6352].

[34] NPLQCD Collaboration, S. Beane et. al., Phys.Rev. D85 (2012) 034505, [arXiv: 1107.5023 ].

[35] G. Colangelo, J. Gasser, and H. Leutwyler, Nucl.Phys. B603 (2001) 125-179, [hep-ph/ 0103088 ].

[36] C. Helmes, C. Jost, B. Knippschild, L. Liu, C. Urbach, et. al., arXiv:1412 . 0408.

[37] C. Morningstar, J. Bulava, J. Foley, K. J. Juge, D. Lenkner, et. al., Phys.Rev. D83 (2011) 114505, [arXiv:1104.3870].

[38] PACS-CS Collaboration, S. Aoki et. al., Phys.Rev. D84 (2011) 094505, [arXiv: 1106.5365 ].

[39] J. Nagata, S. Muroya, and A. Nakamura, Phys.Rev. C80 (2009) 045203, [arXiv: 0812.1753 ].

[40] Z. Fu, Phys.Rev. D85 (2012) 074501, [arXiv:1110.1422].

[41] C. Lang, L. Leskovec, D. Mohler, and S. Prelovsek, Phys.Rev. D86 (2012) 054508, [arXiv:1207.3204].

[42] P. Buettiker, S. Descotes-Genon, and B. Moussallam, Eur.Phys.J. C33 (2004) 409-432, [hep-ph/0310283].

[43] NPLQCD Collaboration, S. R. Beane, P. F. Bedaque, T. C. Luu, K. Orginos, E. Pallante, et. al., Phys.Rev. D74 (2006) 114503, [hep-lat/ 0607036$].$

[44] RBC-UKQCD Collaboration, T. Janowski.

https://indico.bnl.gov/getFile.py/access?contribId=130\&sessionId=2\&resId=0\&materialId=slides\&confId=736.

[45] Hadron Spectrum Collaboration, D. J. Wilson, J. J. Dudek, R. G. Edwards, and C. E. Thomas, arXiv:1411.2004.

[46] M. Lüscher and U. Wolff, Nucl. Phys. B339 (1990) 222-252.

[47] CP-PACS Collaboration, S. Aoki et. al., Phys.Rev. D76 (2007) 094506, [arXiv: 0708 . 3705 ].

[48] ETM Collaboration, X. Feng, K. Jansen, and D. B. Renner, Phys.Rev. D83 (2011) 094505, [arXiv:1011.5288].

[49] C. Lang, D. Mohler, S. Prelovsek, and M. Vidmar, Phys.Rev. D84 (2011), no. 5054503 , [arXiv: 1105.5636 ]. [Erratum-ibid. D89 (2014), no. 5 059903].

[50] C. Pelissier and A. Alexandru, Phys.Rev. D87 (2013) 014503, [arXiv: 1211.0092 ].

[51] Hadron Spectrum Collaboration, J. J. Dudek, R. G. Edwards, and C. E. Thomas, Phys.Rev. D87 (2013), no. 3 034505, [arXiv:1212.0830]. 
[52] B. Fahy, J. Bulava, B. Hörz, K. J. Juge, C. Morningstar, et. al., arXiv: 1410.8843.

[53] BMW Collaboration, T. Metivet, arXiv:1410.8447.

[54] Z. Fu and K. Fu, Phys.Rev. D86 (2012) 094507, [arXiv:1209. 0350].

[55] S. Prelovsek, L. Leskovec, C. Lang, and D. Mohler, Phys.Rev. D88 (2013), no. 5 054508, [arXiv:1307.0736].

[56] Hadron Spectrum, J. J. Dudek, R. G. Edwards, C. E. Thomas, and D. J. Wilson, Phys.Rev.Lett. 113 (2014), no. 18 182001, [arXiv:1406.4158].

[57] S. Ozaki and S. Sasaki, Phys.Rev. D87 (2013) 014506, [arXiv: 1211. 5512].

[58] V. Verduci and C. B. Lang, arXiv:1412.0701.

[59] J. Giedt and D. Howarth, arXiv:1405.4524.

[60] M. Wakayama, T. Kunihiro, S. Muroya, A. Nakamura, C. Nonaka, et. al., arXiv:1412.3909.

[61] A. Abdel-Rehim, C. Alexandrou, J. Berlin, M. D. Brida, M. Gravina, et. al., arXiv: 1410.8757.

[62] N. Ishii, S. Aoki, and T. Hatsuda, Phys. Rev. Lett. 99 (2007) 022001, [nucl-th/ 0611096 ].

[63] S. Aoki, T. Hatsuda, and N. Ishii, Prog.Theor.Phys. 123 (2010) 89-128, [arXiv: 0909.5585 ].

[64] C. D. Lin, G. Martinelli, C. T. Sachrajda, and M. Testa, Nucl.Phys. B619 (2001) 467-498, [hep-lat/0104006].

[65] J. Balog, M. Niedermaier, F. Niedermayer, A. Patrascioiu, E. Seiler, et. al., Phys.Rev. D60 (1999) 094508, [hep-lat/9903036].

[66] CP-PACS Collaboration, S. Aoki et. al., Phys. Rev. D71 (2005) 094504, [hep-lat / 0503025$].$

[67] HALQCD Collaboration, N. Ishii et. al., Phys.Lett. B712 (2012) 437-441, [arXiv: 1203.3642 ].

[68] HALQCD Collaboration, N. Ishii, PoS CD12 (2013) 025.

[69] HALQCD Collaboration, M. Yamada, PoS LATTICE2013 (2014) 232.

[70] HALQCD Collaboration, T. Doi et. al., Prog.Theor.Phys. 127 (2012) 723-738, [arXiv:1106.2276].

[71] T. Kurth, N. Ishii, T. Doi, S. Aoki, and T. Hatsuda, JHEP 1312 (2013) 015, [arXiv: 1305.4462 ].

[72] HALQCD Collaboration, T. Inoue et. al., Phys.Rev.Lett. 106 (2011) 162002, [arXiv:1012.5928].

[73] HALQCD Collaboration, T. Inoue et. al., Nucl. Phys. A881 (2012) 28-43, [arXiv:1112.592 6].

[74] J. Green, A. Francis, P. Junnarkar, C. Miao, T. Rae, et. al., arXiv:1411.1643.

[75] A. Francis, J. Green, P. Junnarkar, C. Miao, T. Rae, and H. Wittig. https://indico.bnl.gov/getFile.py/access?contribId=28\&sessionId=9\&resId=0\&materialId=slides\&confId=934.

[76] M. Fukugita, Y. Kuramashi, M. Okawa, H. Mino, and A. Ukawa, Phys. Rev. D52 (1995) 3003-3023, [hep-lat/9501024].

[77] S. R. Beane, P. F. Bedaque, K. Orginos, and M. J. Savage, Phys. Rev. Lett. 97 (2006) 012001, [hep-lat/0602010].

[78] NPLQCD Collaboration, S. Beane et. al., Phys. Rev. D85 (2012) 054511, [arXiv:1109.2889].

[79] T. T. Takahashi and Y. Kanada-En’yo, Phys.Rev. D82 (2010) 094506, [arXiv: 0912.0691 ]. 
[80] K. Murano, N. Ishii, S. Aoki, and T. Hatsuda, Prog.Theor.Phys. 125 (2011) 1225-1240, [arXiv:1103.0619].

[81] HALQCD Collaboration, T. Doi, PoS LATTICE2013 (2014) 226, [arXiv: 1311.2697 ].

[82] A. Walker-Loud, PoS LATTICE2013 (2014) 013, [arXiv : 1401 . 8259].

[83] PACS-CS Collaboration, T. Yamazaki, Y. Kuramashi, and A. Ukawa, Phys.Rev. D81 (2010) 111504, [arXiv:0912.1383].

[84] T. Doi and M. G. Endres, Comput.Phys.Commun. 184 (2013) 117, [arXiv: 1205.0585 ].

[85] W. Detmold and K. Orginos, Phys.Rev. D87 (2013), no. 11 114512, [arXiv: 1207.1452 ].

[86] J. Günther, B. C. Toth, and L. Varnhorst, Phys.Rev. D87 (2013), no. 9094513 , [arXiv:1301.4895].

[87] P. Vachaspati and W. Detmold, arXiv:1411.3691.

[88] NPLQCD Collaboration, S. Beane, E. Chang, S. Cohen, W. Detmold, H. Lin, et. al., Phys.Rev.Lett. 113 (2014), no. 25 252001, [arXiv:1409.3556].

[89] NPLQCD Collaboration, S. Beane, E. Chang, S. Cohen, W. Detmold, H. W. Lin, et. al., arXiv:1410.7069.

[90] HALQCD Collaboration, F. Etminan et. al., Nucl.Phys. A928 (2014) 89-98, [arXiv:1403.7284].

[91] W. Detmold, M. McCullough, and A. Pochinsky, Phys.Rev. D90 (2014), no. 11114506 , [arXiv:1406.4116]. 